\title{
TOPOLOGICAL CLASSIFICATION OF POLYNOMIAL COMPLEX DIFFERENTIAL EQUATIONS WITH ALL THE CRITICAL POINTS OF CENTER TYPE
}

\author{
M.J. ÁlVAREZ, A. GASUlL, AND R. PROHENS
}

\begin{abstract}
In this paper we study the global phase portrait of complex polynomial differential equations of degree $n$ of the form $\dot{z}=f(z)$, having all their critical points of center type. We give the exact number of topologically different phase portraits on the Poincaré disk when $n \leq 6$ and, in the remaining cases, an upper bound for this number which only depends on $n$.
\end{abstract}

\section{INTRODUCTION AND MAIN RESULT}

In this work we deal with the equation

$$
\dot{z}:=\frac{d z}{d t}=f(z), \quad t \in \mathbb{R}, \quad z \in \mathbb{C},
$$

where $f$ is a complex polynomial of degree $n \geq 1$.

Many characteristics of equation (1.1) are well-known. It presents only three type of finite simple critical points (focus, center and node) all of index +1 , and the centers are always isochronous. Moreover, this type of equations can not have limit cycles, see for instance $[5,11,15,16]$. Regarding the points at infinity in the Poincaré compactification, it is proved that they have exactly $n-1$ couples of saddle points (see $[1,11]$ ) .

In the previous paper [1] the authors explored the relationship between the geometric distribution of the critical points and their type, paying special attention to the case in which the critical points were centers.

In this paper we are going to assume that all the critical points are centers and we will be concerned with the global phase portrait of equation (1.1) on the Poincaré disk. By the results of [21] (that we recall in Theorem 2.3), it turns out that this problem is equivalent to a separatrix configurations problem. In our context, the only separatrices will be the ones of the saddle points at infinity. The problem of studying the number of different phase portraits of equation (1.1), without the assumption of having all the critical points of center type, is also considered in the recent papers [4, 9].

The objective of the present work is to give an upper bound, depending only on $n$, for the number of topologically different global phase portraits

2000 Mathematics Subject Classification. Primary 34C05, Secondary: 34A34, 32A10, $37 \mathrm{C} 10$.

Key words and phrases. Polynomial vector field, Holomorphic vector field, Phase portrait, Graph.

The first and second authors are supported by grants MTM2005-06098-C02-1 and 2005SGR-00550. 
that equation (1.1) can have, when all its critical points are centers. Moreover, for low degrees, we give a sharp upper bound, in the sense that all possible topological configurations are realizable. We prove:

Theorem A. Consider equation (1.1) and assume that all its $n$ critical points are centers. Let $N(n)$ be the number of topologically different phase portraits on the Poincaré disk of this equation satisfying this condition. Then

$$
N(1)=N(2)=N(3)=1, N(4)=2, N(5)=3, N(6)=6 .
$$

Proposition B. Let $N(n)$ be defined as in Theorem A. Then $N(7) \leq 12$, $N(8) \leq 26$ and for $n \geq 9$,

$$
N(n) \leq M(n):=\left[\frac{G(n)-24}{7}\right]+5,
$$

where $[x]$ denotes the integer part of $x$ and the function $G$ is defined by the following recursion formula

$$
G(m)=\sum_{k=1}^{m-1} G(m-k) G(k),
$$

with $G(1)=1$.

Remark 1.1. For small $n$ the upper bounds given in the above proposition are

$$
M(9)=205, M(10)=696, M(11)=2401, \ldots
$$

A case by case study could be used to improve them. See also Remark 3.1.

We end this introduction by explaining our motivations to study the problem considered in this paper.

The Hilbert's 16th Problem asks about the existence and determination of the maximum number of limit cycles, $\mathcal{H}(n)$, that planar real polynomial differential equations of degree $n$ can have, see $[18,20]$. One of the approaches to get lower bounds of $\mathcal{H}(n)$ consists in perturbing planar systems having several continua of periodic orbits and then study how many of them remain as limit cycles of the perturbed system. The first step in this direction, which is called weak, tangential or infinitesimal Hilbert's 16th Problem, consists in taking the unperturbed systems to be a polynomial Hamiltonian system and then studying the first order perturbations, through the so called Abelian integrals, see $[7,18,20]$. Another natural problem in this context is:

Perturbation of holomorphic systems problem: Consider the real planar polynomial equations of the form

$$
\dot{z}=f(z)+\varepsilon h(z, \bar{z}),
$$

where $f$ and $h$ are complex polynomials of degree $n, \varepsilon$ is a small real parameter and $\dot{z}=f(z)$ has at least one critical point of center type. Then study, in terms of n, how many many limit cycles bifurcate from the periodic orbits of the unperturbed equation.

Some partial results for $n=2,3$ are done in $[6,12,13,19]$.

It is clear that the systems $\dot{z}=f(z)$ studied in this paper are the natural candidates in (1.3) to give the maximum number of limit cycles after perturbation. 
Of course, another interesting problem consists in studying the same question when the degrees of $f$ and $h$ are different. The easiest unperturbed case $n=1, \dot{z}=i z$, which is precisely the only intersection between the Hamiltonian and the holomorphic centers is considered in [17]. For some results for the cases $n=2,3$, see again $[12,19]$.

The systems considered in our paper are also useful as starting points for studying the number of complex isolated periodic orbits of non-autonomous complex differential equations of Abel type, see [8].

\section{Preliminary and Related Results}

In this section we recall several results concerning equation (1.1). We also explain the relationship between the problem we are studying and planar graphs.

The first result classifies the behavior of the solutions of the differential equation $\dot{z}=f(z)$ near its finite and infinite critical points, see for instance $[5,11,15,16]$. We point out that, to represent the infinity of the complex plane we use the Poincaré compactification, see for instance [10, Chap. 5] or [22, Chap. 3.10]. It involves identifying the plane with one of the two hemispheres of the sphere by a central projection and consequently the infinity is represented by the equator. By projecting, for instance, the north hemisphere into a disk, we can represent the flow of the plane in a disk, called the Poincaré disk, where now the infinity is its boundary, $\mathbb{S}^{1}$. Observe that if $p \in \mathbb{S}^{1}$ is an infinite critical point then $-p$ also it is.

Theorem 2.1. Consider a polynomial differential equation $\dot{z}=f(z)$ of degree $n$. Let $z=z_{0}$ be one of its finite critical points.

(a) If $z=z_{0}$ is a simple zero of $f$ then:

(I) It is an isochronous center when $f^{\prime}\left(z_{0}\right) \in i \mathbb{R}$,

(II) It is a node when $f^{\prime}\left(z_{0}\right) \in \mathbb{R}$,

(III) It is a focus when $\operatorname{Re}\left(f^{\prime}\left(z_{0}\right)\right) \operatorname{Im}\left(f^{\prime}\left(z_{0}\right)\right) \neq 0$.

(b) If $z=z_{0}$ is a zero of multiplicity $m$ of $f$ then it is the union of $2(m-1)$ elliptic sectors.

(c) At infinity it has exactly $n-1$ couples of critical points, all of them being hyperbolic saddles. Moreover they are uniformly distributed in the boundary of the disk, being $\pi /(n-1)$ the angle between each two consecutive saddles.

Remark 2.2. Observe that $z_{0}$ is a center of the equation $\dot{z}=f(z)$ if and only if $z_{0}$ is a node of equation $\dot{z}=i f(z)$. Hence, if an equation $\dot{x}=f(z)$ has all its critical points of center type, then the corresponding equation $\dot{z}=i f(z)$ has all its critical points of nodal type.

We recall that a separatrix of equation (1.1) is an orbit which is either a critical point or a trajectory which separates two hyperbolic sectors. In [21] it was proved that the set formed by all separatrices of a differential equation is closed. For a given equation, its separatrix configuration is the union of all separatrices together with a representative orbit from each canonical region (in our case, one closed orbit surrounding each center). Two separatrix configurations are said to be topologically equivalent if there is an orientation preserving homeomorphism which maps the trajectories in 
the first one into the trajectories in the second. In [21], it was also proved that the global phase portrait of an equation is univocally determined by its separatrix configuration. Here we state the result.

Theorem 2.3 ([21]). Suppose $\phi_{1}$ and $\phi_{2}$ are continuous flows on a 2manifold $\mathcal{M}$, with isolated critical points. Then they are equivalent if and only if their separatrix configurations are equivalent.

We want to bound the number of topologically different phase portraits of equation (1.1), with all the critical points being of center type. Observe that inside each region defined by the heteroclinic connections of the infinite saddles there has to be exactly one center. Hence, when we refer to the separatrix configuration we will only pay attention to the infinite saddle connections.

According to the results in [21], in order to count the number of different phase portraits of equation (1.1) we have to count the number of different possible combinations for saddle connections between the $2 n-2$ critical points on the boundary of the Poincare disk. Hence our problem turns out to be a combinatorial problem. We can consider the infinite saddle points as vertices of a planar graph (planar because separatrices can not intersect) and their separatrices as edges. In this context, each vertex has degree 3, but two edges of each point are fixed (the two infinite separatrices), see some examples in Figure 1.

The problem of counting the number of planar graphs with a fixed number of vertices and separatrices is not a trivial one. There is a lot of literature on the subject and in [14] it was proved an asymptotic formula. However, this formula does not fit our case because it does not consider all our restrictions. Moreover, as we are only concerned with the topologically different phase portraits, we would be much more interested in counting the so-called unlabeled planar graphs, which are the ones for which the vertices are indistinguishable. Then, we have to count the number of isomorphism classes of the graphs with a fixed number of vertices and edges. This problem turns out to be more difficult than the one distinguishing vertices, because of the symmetries that the graph can present.

In [3] there is proved an asymptotic formula for the number of unlabeled planar graphs with $m$ vertices. This result affirms that there are at most $2^{\alpha m}$ graphs, with $\alpha \approx 4.9098$, for $m$ big enough.

This result does not take into account the fact that the infinite critical points are located on a circumference and all the separatrices lie inside it. The graphs with these restrictions are called (unlabeled, if we do not distinguish vertices) outerplanar graphs and have also been extensively studied.

Recently, it has been proved in [2] an asymptotic formula for the number of unlabeled outerplanar graphs, $g_{m}$, with $m$ vertices, $g_{m} \sim g m^{-5 / 2} \rho^{-m}$ where $\rho^{-1} \approx 7.50360$ and $g \approx 0.00909941$.

Both formulas are asymptotic (so not useful for small number of vertices) and not adapted to our concrete problem. So in next section we count the number of topologically different phase portraits for small degrees of $f$ and we develop a general upper bound for any degree which takes into account all the restrictions of our problem. 


\section{Proof of the main Results}

3.1. Proof of Theorem A. By using Theorem 2.3 it is easy to see that for $n \leq 6$ the values of $N(n)$ given in the statement of the theorem are upper bounds for their actual values. To end the proof it only remains to show that all these topological possibilities are realizable. So, for each one of them we will give a differential equation of the form (1.1) having the corresponding topological configuration as its phase portrait.

For $n=1$, there is only one finite center and there are no saddles at infinity. Hence, there is only one topological possibility, which is realized by $\dot{z}=i z$, for instance. Then, $N(1)=1$.

For $n=2$ (respectively, $n=3$ ) there is again only one possibility of configuration of separatrices joining the infinite critical points. Hence, any equation (1.1) of degree 2 (respectively 3), having all the critical points of center type, has this topological configuration as its phase portrait. For instance we can take $\dot{z}=i z(z-1)$ (respectively, $\dot{z}=i z(z-1)(z-2)$ ).

For $n=4$, there are two topological possibilities, as it can be seen in Figure 1.

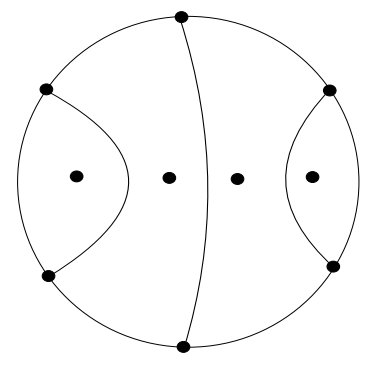

(a)

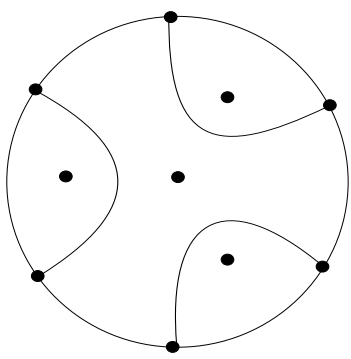

(b)

FiguRE 1. Infinite saddle connexions of the two different phase portraits of equation (1.1) when $n=4$.

Next, we give differential equations having these graphs as their phase portraits. Concerning Figure 1(a), consider equation

$$
\dot{z}=i z(z-1)(z-2)(z-4) \text {. }
$$

It is invariant under the change of variables $(z, t) \rightarrow(\bar{z},-t)$ and, consequently, its phase portrait presents a symmetry with respect the axis $\operatorname{Im}(z)=0$. Due to this symmetry, the only way of joining the six infinite critical points is the one plotted in Figure 1(a).

Concerning Figure 1(b), consider equation

$$
\dot{z}=z\left(z^{3}-i / 3\right) \text {. }
$$

It has three of its critical points located on the vertices of an equilateral triangle and the fourth one on its orthocenter, all of them being centers. Moreover, it is invariant under a rotation of $\pi / 3$ radians and all them are centers. Because of that, its phase portrait is the one depicted in Figure 1(b).

For $n=5$, there are three topological possibilities to join the eight infinite critical points. See Figure 2. 


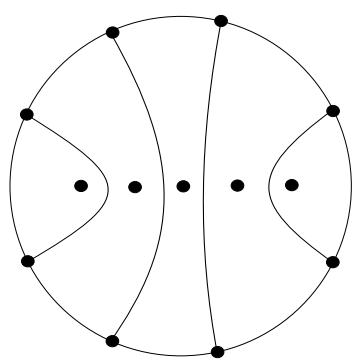

(a)

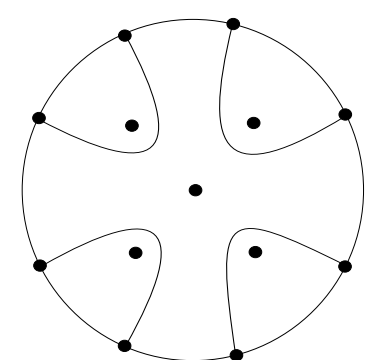

(b)

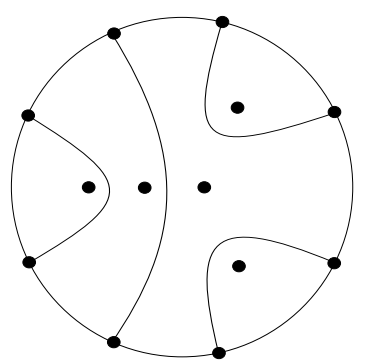

(c)

FiguRE 2. The three topologically different phase portraits for $n=5$.

Using symmetry arguments, as before, we can conclude that each one corresponds with one of the following equations,

$$
\begin{aligned}
\dot{z} & =i z(z-1)(z-2)(z-4)(z-7), \\
\dot{z} & =z\left(z^{4}-i / 4\right), \\
\dot{z} & =i z(z-1)(z-2)(z-3+i)(z-3-i),
\end{aligned}
$$

in the following order: Figure 2(a) is fulfilled by equation (3.1), Figure 2(b) by equation (3.2) and Figure 2(c) by equation (3.3).

For $n=6$, there are six possible topological configuration of separatrices. See Figure 3. Using analogous symmetry arguments, as before, and Theo-

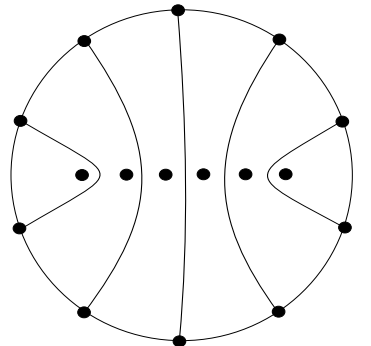

(a)

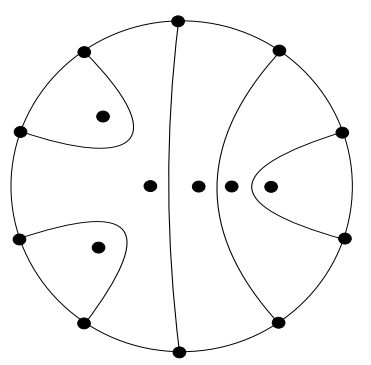

(d)

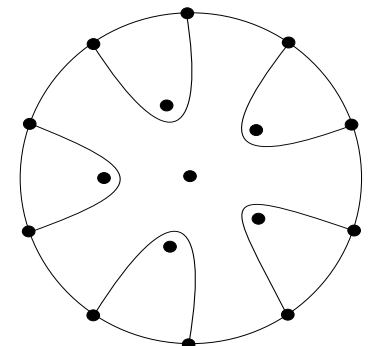

(b)

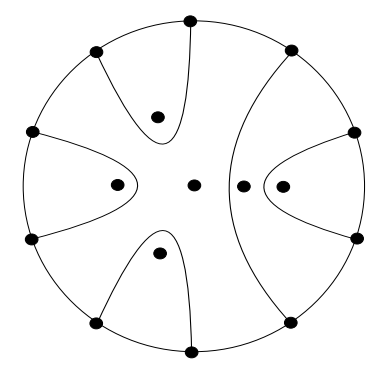

(e)

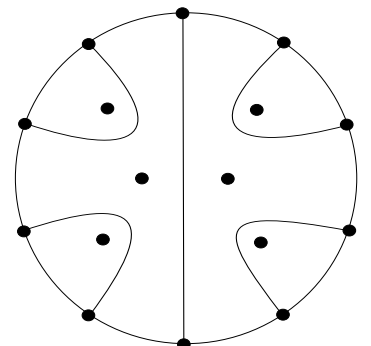

(c)

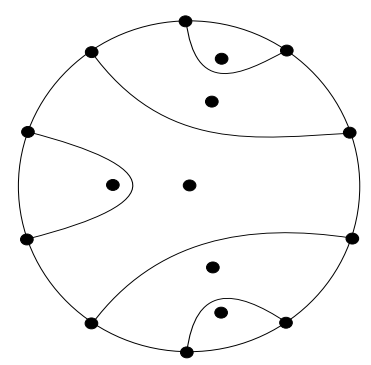

(f)

FiguRE 3. The six topological different phase portraits for $n=6$.

rem 2.1, we get that three equations that fulfill the phase portraits plotted 
in Figure 3, (a), (b) and (c) are,

$$
\begin{aligned}
& \dot{z}=i z(z-1)(z-2)(z-4)(z+4)(z-8), \\
& \dot{z}=z\left(z^{5}-i / 5\right), \\
& \dot{z}=i(z-\sqrt{6})(z+\sqrt{6})\left(z-z_{3}\right)\left(z-\bar{z}_{3}\right)\left(z+z_{3}\right)\left(z+\bar{z}_{3}\right),
\end{aligned}
$$

with $z_{3}=3+i$, respectively.

Observe that equations (3.4) and (3.6) are invariant under the change of variables $(z, t) \rightarrow(\bar{z},-t)$ and then their phase portraits are symmetric with respect to the axis $\operatorname{Im}(z)=0$. Moreover, equation (3.6) is also invariant under $(z, t) \rightarrow(-\bar{z},-t)$. Because of that, together with the location of the critical points, we can conclude that they fulfill the corresponding phase portraits (a), (b) and (c).

To prove that the configurations appearing in Figure 3(d) and 3(e) are realizable, consider next equation:

$$
\dot{z}=f(z)=i z(z-1)(z-2)(z-3)(z-u+i)(z-u-i) .
$$

By using again Theorem 2.1 we get that this equation has 4 centers located at $z=0,1,2,3$, for all values of the parameter $u$ and it has two more centers at $z=u \pm i$ if $u \in\{(3 \pm \sqrt{17 \pm 4 \sqrt{14}}) / 2,(3 \pm \sqrt{17 \mp 4 \sqrt{14}}) / 2\}$. Moreover, it is invariant under the change of variables $(z, t) \rightarrow(\bar{z},-t)$ and, because of the distribution of the critical points, the phase portraits that this equation can have are either (d) or (e) of Figure 3.

Let us prove that when $u=(3-\sqrt{17+4 \sqrt{14}}) / 2$, the corresponding phase portrait for equation (3.7) is Figure 3(d). To do this, we compute the contact points of the flow of the equation (3.7) with the segment $\{u+i y,-1 \leq y \leq 1\}$ joining the two non-real centers. These contact points are given by the roots for $y \in(-1,1)$ of the equation

$$
\operatorname{Re}(f(u+i y))=2 \sqrt{17+4 \sqrt{14}}\left(y^{2}-3-\sqrt{14}\right) y(y-1)(y+1)=0 .
$$

We note that there is only one contact point on this line between the two critical points (which is $y=0$ ). If the phase portrait was the one given in Figure 3(e), then there should be at least three contact points. See Figure 4.

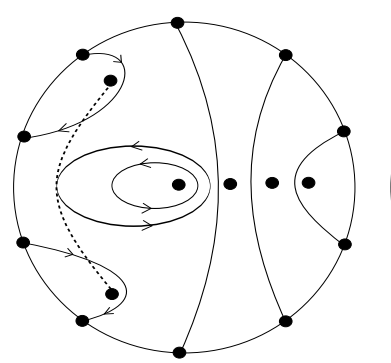

(d)

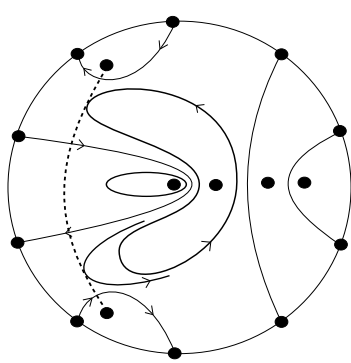

(e)

Figure 4. Contact points of the flow of equation (3.7), over the segment joining the two non-real centers, on $\operatorname{Re}(z)=u$.

Hence, it turns out that the phase portrait must be the one plotted in Figure 3(d). 
In order to prove that configuration (e) in Figure 3 is also realizable we consider again equation (3.7), but now with $u=(3-\sqrt{17-4 \sqrt{14}}) / 2$.

In this case we compute the contact points of flow of the vector field with the segment that joins the centers $z=u+i$ and $z=1,\{x+(x-1) i /(u-$ $1), u \leq x \leq 1\}$. These are given by the real roots of

$$
\operatorname{Re}\left(f\left(x+\frac{x-1}{u-1} i\right)\right)+(1-u) \operatorname{Im}\left(f\left(x+\frac{x-1}{u-1} i\right)\right)=(x-1)(x-u) P_{4}(x)=0,
$$

where $P_{4}(x)$ is a polynomial without real roots in the interval $(u, 1)$. Hence, there is no contact point of the flow with this segment. We note that, if the phase portrait of equation (3.7) was the one plotted on Figure 3(d), then there should be at least one contact point. See Figure 5 .

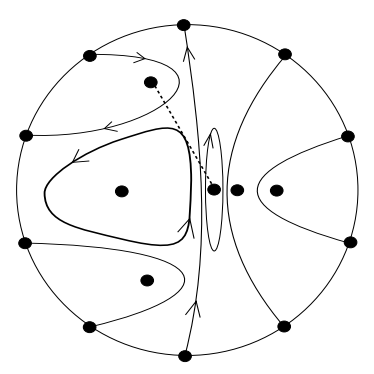

(d)

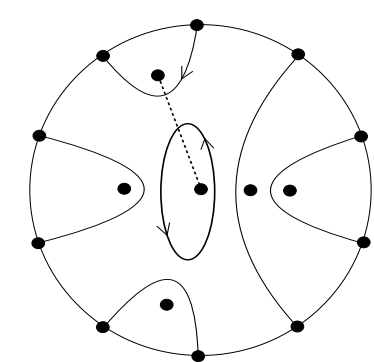

(e)

Figure 5. Contact points of the flow of equation (3.7), over the segment joining $z=u+i$ and $z=1$.

Consequently, the phase portrait associated to equation (3.7) is the one in Figure 3(e).

Finally we prove that configuration (f) in Figure 3 is also realizable. Consider equation,

$$
\dot{z}=f(z)=i(z-a)(z-b)\left(z-z_{3}\right)\left(z-\bar{z}_{3}\right)\left(z-z_{4}\right)\left(z-\bar{z}_{4}\right),
$$

with $z_{3}=1+\tan (2 \pi / 5) i, z_{4}=10 z_{3}$ and $a, b \in \mathbb{R}, a \neq b$. We note that, we have that $\operatorname{Re}\left(f^{\prime}(a)\right)=\operatorname{Re}\left(f^{\prime}(b)\right)=0$ and hence these critical points are centers.

It is not difficult to see that if $f^{\prime}\left(z_{3}\right), f^{\prime}\left(z_{4}\right) \in i \mathbb{R}$, then the same holds for $\bar{z}_{3}$ and $\bar{z}_{4}$. Hence we only need to impose two conditions on $a$ and $b$ to get six centers in equation (3.8). Here we want to point out that this situation can also be interpreted in the light of [1, Prop. 2.7]. In that result a differential equation of the form (1.1) having $s$ centers (or nodes) on a straight line and the other $n-s$ critical points, symmetric two by two with respect to this line, is studied.

In our case the center conditions are:

$$
\begin{aligned}
\operatorname{Re}\left(f^{\prime}\left(z_{3}\right)\right)= & -18\left(\alpha\left(9+11 \alpha^{2}\right) a b+\alpha\left(-9-13 \alpha^{2}\right) a+\alpha\left(-9-13 \alpha^{2}\right) b+\right. \\
& \left.\alpha\left(9+6 \alpha^{2}-11 \alpha^{4}\right)\right)=0, \\
\operatorname{Re}\left(f^{\prime}\left(z_{4}\right)\right)= & 180\left(\alpha\left(-9+11 \alpha^{2}\right) a b+\alpha\left(90-310 \alpha^{2}\right) a+\alpha\left(90-310 \alpha^{2}\right) b+\right. \\
& \left.\alpha\left(-900+6000 \alpha^{2}-1100 \alpha^{4}\right)\right)=0,
\end{aligned}
$$


where $\alpha=\tan (2 \pi / 5)$. By doing the resultant of these two polynomials on two variables with respect to $b$ one gets a polynomial of degree 4 only depending on $a$. Hence, it is possible to solve analytically the above system. For instance one of its solutions is $a=a^{*} \approx 0.612777$ and $b=b^{*} \approx-15.962837$.

Now, we are going to prove that the phase portrait of equation (3.8) when $a=a^{*}$ and $b=b^{*}$ is the one in Figure 3(f). First of all, we observe that the equation is invariant under the change of variables $(z, t) \rightarrow(\bar{z},-t)$ and consequently the phase portrait is symmetric with respect to the line $\operatorname{Im}(z)=0$, but it is not with respect to $\operatorname{Re}(z)=0$. This fact, together with the location of the finite critical points, implies that the phase portrait of the equation can not be the ones depicted in Figure 3(a), (c), (d) or (e). Then, it can only be the phase portrait in (b) or in (f).

To decide which phase portrait is the right one, we use the same technique that in the previous cases. Here we prove that there are no contact points between the flow of the differential equation and the segment of the straight line $y=\tan \left(\frac{2 \pi}{5}\right) x$, between the two critical points located on it. Hence its phase portrait can not be the one in Figure 3(b) and it must be the one shown in Figure 3(f), as we wanted to see.

3.2. Proof of Proposition B. The proof that when $n=7$ (respectively $n=8$ ) there are at most 12 (respectively 26) topologically different phase portraits on the Poincaré disk is straightforward by using Theorem 2.3 and a tedious case by case study.

To get a general upper bound for $N(n)$ when $n \geq 9$, we want to count how many different topological possibilities are there to join up $2 n-2$ points, located on a circumference, two by two and without intersections between the edges and taking into account the alternating stabilities of separatrices of the infinite saddles.

To do this, we proceed in two steps. First, we label the infinite saddle points from $p_{1}$ to $p_{2 n-2}$ and, then, we count how many possibilities are. Let $G(n)$ be this number. Next, we will deduct those phase portraits that have been counted multiple times. These deductions will only take into account repetitions because of rotations.

Let us start the first step. If we join $p_{1}$ to $p_{k}$ then we have reduced the problem of joining $2 n-2$ points to two simpler problems: one with $k-2$ points and the other with $2 n-2-k$ points. Each one of these problems can be reduced to two simpler ones and we can solve the original problem in a recursive way.

Observe that we can only join the point $p_{i}, i$ odd, to $p_{j}, j$ even, because of the alternating stabilities of the separatrices of consecutive saddles.

As we can join $p_{1}$ to any $p_{k}, k$ even, then we have to sum all the possibilities:

$$
G(n)=\sum_{k=1}^{n-1} G(n-k) G(k) .
$$

Moreover, the base case is obviously $G(1)=1$ because if there are no critical points at infinity, then there is only one possibility for the graph. It is also clear that $G(2)=1$. 
Let us now start the second step of refining the formula, by taking into account that in this process there are topologically equal phase portraits that have been counted multiple times. If we have constructed a phase portrait, we can rotate it by an angle $\beta=\pi /(n-1)$ radians, (recall, that by Theorem 2.1, $\beta$ is the angle between two infinite critical points) and with this rotation, if the point $p_{i}$ is joined to the point $p_{j}$ now the point $p_{i+1}$ will be joined to $p_{j+1}$, where we take the subindexes modulus $2 n-2$. With this operation we will always obtain a different separatrix configuration. But in our formula we have counted them as different phase portraits while they are topologically equivalent. Then, each phase portrait is counted, at least, twice (see Figure 6).
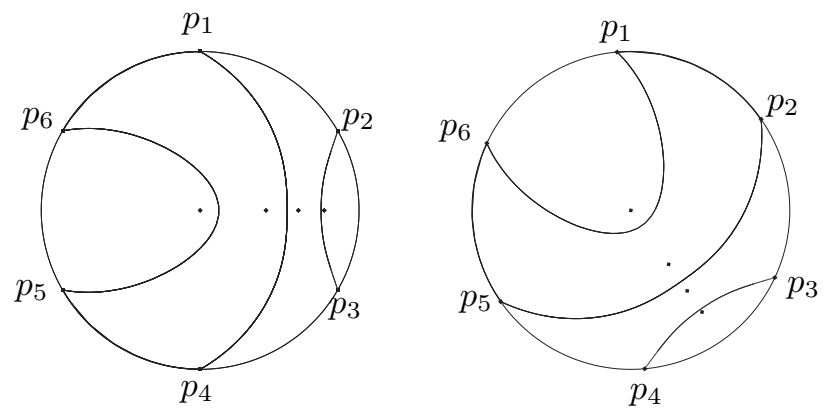

FiguRE $6 . \quad$ Rotating a phase portrait an angle $\beta=\pi /(n-1)$ gives rise to another that is topologically equivalent to the original one. In the figure $n=4$.

We can do the same reasoning and make another rotation of $\beta$ radians, i.e., if the point $p_{i}$ is originally joined to the point $p_{j}$ now the point $p_{i+2}$ will be joined to $p_{j+2}$. But now we have to take into account that it is possible that we get exactly the original phase portrait. It would be the case in which every point $p_{i}$ is joined to $p_{i+1}$, (the one depicted in Figure $7(\mathrm{a})$ ) that is counted exactly twice.

By doing this reasoning we can deduct some phase portrait that are counted several times. We claim that, for any $n \geq 7$, among all the $G(n)$ phase portraits obtained by the above procedure, all except at most five of them, are repeated at least seven times. The five phase portraits that are counted less than seven times appear when $n-1=\dot{6}$. In Figure 7 we give them when $n=13$. The obvious generalization of the ones given in (b) of the figure appear when $n-1=\dot{2}$ while the corresponding to the ones given in (c)-(d) and (e) appear when $n-1=\dot{3}$.

Let us prove now the claim. Suppose there exists one phase portrait that is counted exactly three times. Suppose also that in this phase portrait $p_{1}$ is joined to $p_{k}$ and $p_{2 n-4}$ is joined to $p_{j}$. As it is counted exactly three times, when rotating it by an angle of $3 \beta$ radians we will recover the same phase portrait. After this rotation, the point $p_{4}$ will be joined to $p_{k+3}$ and $p_{2 n-1}=$ $p_{1}$ will be joined to $p_{j+3}$. But as the rotated phase portrait has to be exactly the original one then $j+3=k \bmod (2 n-2)$. We can conclude that $k<4$ because otherwise the separatrix joining $p_{1}$ to $p_{j+3}=p_{k}$ will intersect the one connecting $p_{4}$ to $p_{k+3}$ (see Figure 8 ) and this is not possible by the uniqueness 


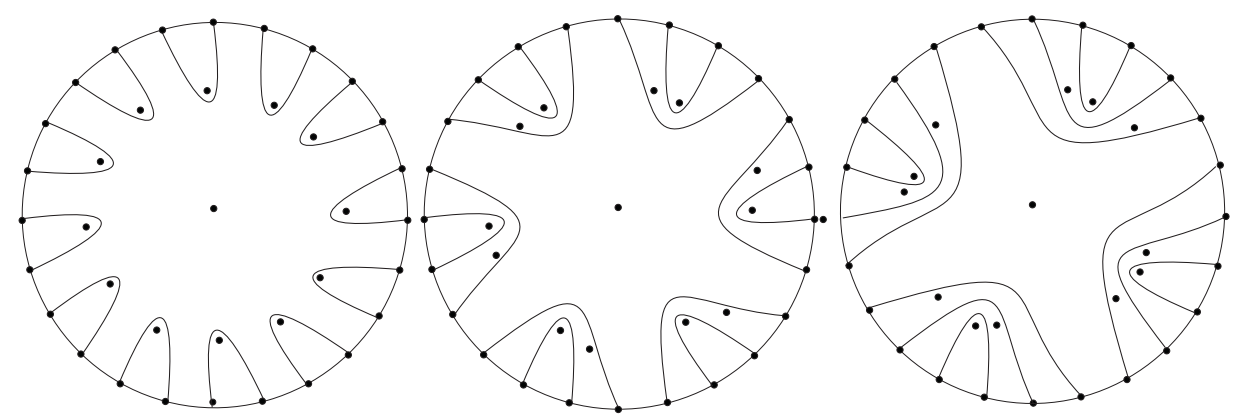

(a)

(b)

(c)

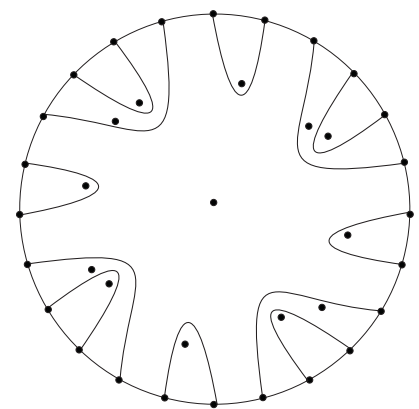

(d)

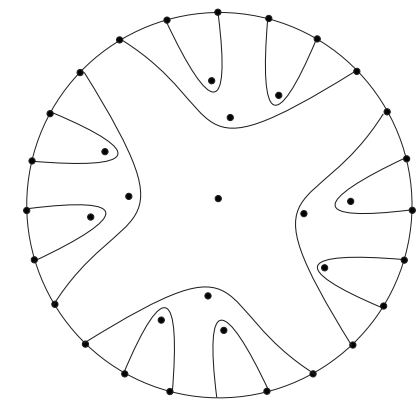

(e)

FIGURE 7. The only 5 phase portraits repeated less than seven times with our procedure. In the figure, $n=13$.
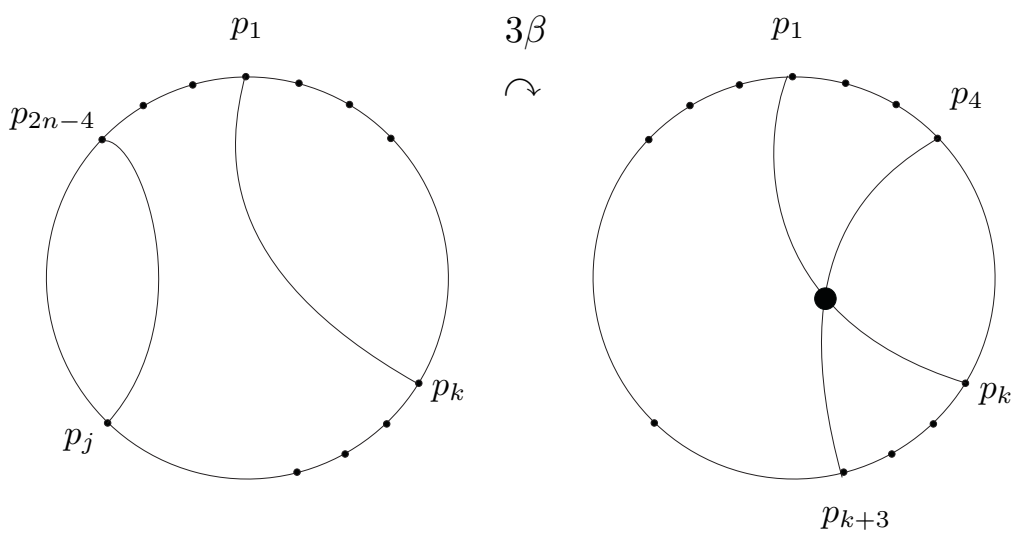

FigURE $8 . \quad$ It is impossible that a phase portrait is repeated exactly three times.

of solutions of the differential equation. Consequently, $p_{1}$ is joined to $p_{2}$ or $p_{3}$. We know that it can not be joined with $p_{3}$ because of the stability of the separatrices. If $p_{1}$ is joined to $p_{2}$, repeating the same arguments for the rest of the infinite critical points we get that $p_{m}$ is connected to $p_{m+1}$ for all $m$ odd and, hence, the phase portrait is counted twice. See Figure 7(a). Consequently, no phase portrait is repeated exactly three times.

Suppose now that there exists a phase portrait counted exactly four times. We will prove that it has to be a natural extension of the one depicted in 
Figure 7(b). Arguing as above we can conclude that the point $p_{1}$ has to be joined to $p_{k}$ with $k<5$. We already know that if it is joined to $p_{2}$ we recover that phase portrait counted only twice and that with $p_{3}$ can not be connected. Thus, it has to be joined to $p_{4}$ and consequently $p_{2}$ has to be connected to $p_{3}$. If we repeat the same arguments for the rest of the points, then we get the result.

By using analogous arguments as above, we will get that no phase portrait is repeated exactly five times, while there are three type of phase portraits counted exactly six times (the natural extensions of the ones in Figure $7(\mathrm{c}),(\mathrm{d})$ and $(\mathrm{e}))$.

After previous refinement, we can prove formula (1.2). Given equation (1.1) and following the procedure described at the beginning of the proof, we get that there are $G(n)$ ways of connecting the $2 n-2$ infinite critical points. Several of these resultant graphs give rise to phase portraits that are topologically equivalent. As we have proved before, all but at most 5 of them are repeated at least seven times. These five phase portraits are repeated, altogether, 24 times (one is repeated twice, one is repeated four times and three are repeated six times, what sums 24). Consequently, we get

$$
N(n) \leq M(n):=\left[\frac{G(n)-24}{7}\right]+5,
$$

as we wanted to prove. Notice that when $n-1 \neq \dot{6}$ we could improve a little bit the above upper bound. For instance when $n-1 \neq \dot{6}$ and $n-1=\dot{2}$ then the upper bound would be $[(G(n)-6) / 7]+2$, which is a little smaller than $M(n)$. Similarly, when $n-1 \neq \dot{3}$ and $n-1 \neq \dot{2}$ the upper bound would be $[(G(n)-2) / 7]+1$.

Remark 3.1. Given any $k \in \mathbb{N}$ and arguing similarly as in the proof of Proposition B it is possible to improve the upper bound given in that result for $n \geq k$. The new upper bounds are of the form

$$
M_{k}(n):=\left[\frac{G(n)-I_{k}}{k}\right]+J_{k},
$$

where $I_{k}$ and $J_{k}$ are integer numbers, being $J_{k}$ the number of phase portraits repeated less than $k$ times by rotations and $I_{k}$ the total number of repetions of these $J_{k}$ phase portraits. They satisfy $J_{k}>I_{k} / k$. For instance taking $k=9$ we get that there are eleven repeated phase portraits: one repeated twice, one repeated four times, three repeated six times and six repeated eight times. Hence, $J_{9}=11$ and $I_{9}=1 \times 2+1 \times 4+3 \times 6+6 \times 8=72$. Consequently,

$$
N(n) \leq M_{9}(n):=\left[\frac{G(n)-72}{9}\right]+11,
$$

because there are five different phase portraits which are invariant after eight rotations of angle $\beta$ that appear when $n-1=\dot{8}$. Notice for instance that $M_{9}(9)=161<M(9)=205$ and $M_{9}(10)=543$. 


\section{REFERENCES}

[1] M.J. Álvarez; A. Gasull; R. Prohens, Configurations of critical points in complex polynomial differential equations, To appear in Nonlinear Anal., Ser. A: Theory Methods \& Applications.

[2] M. Bodirsky; E. Fusy; M. Kang; S. Vigerske, Enumeration and asymptotic properties of unlabeled outerplanar graphs, The Electronic Journal of Combinatorics 14 (2007), R66.

[3] N. Bonichon, C. Gavoille, N. Hanusse, D. Poulalhon and G. Schaeffer, Planar graphs, via well-orderly maps and trees, Graphs and Combinatorics 22 (2006), 185-202.

[4] B. Branner and K. Dias, Classification of complex polynomial vector fields in one complex variable, Preprint 2008.

[5] L. Brickman and E.S. Thomas, Conformal equivalence of analytic flows, J. Differential Equations 25 (1977), 310-324.

[6] C. Chicone and M. Jacobs, Bifurcation of limit cycles from quadratic isochronous, J. Differential Equations 91 (1991), 268-326.

[7] C. Christopher, C. Li, Limit cycles of differential equations. Advanced Courses in Mathematics, CRM Barcelona, Bikhäuser, 2007.

[8] A. Cima, A. Gasull and F. Mañosas, Periodic orbits in complex Abel equations, J. Differential Equations 232 (2007), 314-328.

[9] K. Dias, Enumeration of combinatorial classes of complex polynomial vector fields in the plane, in preparation.

[10] F. Dumortier, J. Llibre and J. C. Artés, Qualitative theory of planar differential systems. Universitext. Springer-Verlag, Berlin, 2006.

[11] A. Garijo, A. Gasull, X. Jarque, Local and global phase portrait of equation $\dot{z}=f(z)$, Discrete Cont. Dyn. Syst. 17 (2007), 309-329.

[12] A. Garijo, A. Gasull and X. Jarque, Simultaneous bifurcation of limit cycles from two nests of periodic orbits, J. Math. Anal. Appl., 341 (2008), 813-824.

[13] A. Gasull, W. Li, Weigu, J. Llibre, Jaume and Z. Zhang, Chebyshev property of complete elliptic integrals and its application to Abelian integrals, Pacific J. Math. 202 (2002), 341-361.

[14] O. Giménez, M. Noy, Asymptotic enumeration and limit laws of planar graphs, http://arxiv.org/abs/math/0501269.

[15] O. Hájek, Notes on meromorphic dynamical systems I, Czech. Mathematical Journal 91 (1966), 14-27.

[16] O. Hájek, Notes on meromorphic dynamical systems II, Czech. Mathematical Journal 91 (1966), 28-35.

[17] I. D. Iliev, The number of limit cycles due to polynomial perturbations of the harmonic oscillator, Math. Proc. Cambridge Philos. Soc. 127 (1999), 317-322.

[18] Yu. Ilyashenko, Centennial history of Hilbert's 16th problem, Bull. Amer. Math. Soc. (N.S.) 39 (2002), 301-354.

[19] C. Li, J. LLibre, Z. Zhang, Linear estimate for the number of zeros of abelian integrals for quadratic isochronous centers, Nonlinearity 13 (2000), 1775-1800.

[20] J. Li, Hilbert's 16th problem and bifurcations of planar polynomial vector fields, Internat. J. Bifur. Chaos Appl. Sci. Engrg. 13 (2003), 47-106.

[21] D. Neumann, Classification of continuous flows on 2-manifolds, Proc. Amer. Math. Soc. 48 (1975), 73-81. 
[22] L. Perko, Differential equations and dynamical systems. Springer, TAM (7), New York, 2001.

Dept. De Matemàtiques i Informàtica, Universitat de les Illes Balears, 07122 Palma de Mallorca, Illes Balears. Spain

E-mail address: chus.alvarez@uib.es

Dept. de Matemàtiques, Universitat Autònoma de Barcelona, Edifici C 08193 Bellaterra, Barcelona. Spain

E-mail address: gasull@mat.uab.cat

Dept. de Matemàtiques i Informàtica, Universitat de les Illes Balears, 07122 Palma de Mallorca, Illes Balears. Spain

E-mail address: rafel.prohens@uib.cat 